\title{
COLISTIN RESISTANCE IN CARBAPENEM-RESISTANT KLEBSIELLA PNEUMONIAE STRAINS
}

\author{
BEENA HOSDURG BHASKAR ${ }^{1}$, SHALINI SHENOY MULKI ${ }^{2}$, SANGEETA JOSHI ${ }^{1}$, RANJEETA ADHIKARI ${ }^{1}$, \\ BHAVANA MALAVALLI VENKATESH ${ }^{1}$
}

${ }^{1}$ Department of Microbiology, Manipal Hospital, Bengaluru, Karnataka, India. ${ }^{2}$ Department of Microbiology, Kasturba Medical College, Mangalore, Karnataka, India. Email: Beenhb@yahoo.co.in

Received: 08 March 2017, Revised and Accepted: 30 May 2017

ABSTRACT

Objective: There is an increasing use of colistin consequent to increase in the infections caused by carbapenem-resistant Klebsiella pneumoniae. The present study was conducted to determine the minimum inhibitory concentration (MIC) of colistin and the resistance pattern of colistin in carbapenem-resistant K. pneumoniae (CRKP) strains in our intensive care unit (ICU).

Methods: Antibiotic susceptibility testing for other antimicrobial agents was done by Kirby-Bauer disk diffusion method. MIC of colistin was determined by agar dilution method. The results of antibiotic susceptibility testing were interpreted as per Clinical Laboratory Standard Institute guidelines 2016 and MIC of colistin were interpreted as per European Committee on Antimicrobial susceptibility testing. The carbapenem resistance was phenotypically detected by modified hodge test and imipenem/imipenem ethylenediaminetetraacetic acid disk method.

Results: Out of $518 \mathrm{~K}$. pneumoniae, 329 were resistant to carbapenems, and 91 isolates showed resistance to colistin. The MIC of colistin ranged between 4 and $>512 \mathrm{ug} / \mathrm{ml}$ and $\mathrm{MIC}_{90}$ was $16 \mathrm{ug} / \mathrm{L}$ and $\mathrm{MIC}_{50}$ was $4 \mathrm{ug} / \mathrm{ml}$. A majority of the colistin-resistant isolates were found in multidisciplinary ICU $(85 / 91)$.

Conclusion: The emergence of colistin-resistant strains is a major problem due to limited treatment options for infections caused by CRKP carbapenemase producing K. pneumoniae. Colistin should not be used alone, combination therapy should be preferred.

Keywords: Colistin, Carbapenemase producing Klebsiella pneumoniae, Minimum inhibitory concentration of colistin.

(C) 2017 The Authors. Published by Innovare Academic Sciences Pvt Ltd. This is an open access article under the CC BY license (http://creativecommons. org/licenses/by/4. 0/) DOI: http://dx.doi.org/10.22159/ajpcr.2017.v10i9.18369

\section{INTRODUCTION}

Enterobacteriaceae are one of the major agents of hospital and community-acquired infections; these cause increased morbidity and mortality, especially with increased resistance rates [1]. Extended spectrum betalactamases (ESBLs) lead to multidrug resistance by transfer between bacteria and are seen in Enterobacteriaceae [2]. Carbapenems are important group of antibiotics used as a last option, especially in ESBL producing multi-drug resistant Enterobacteriaceae [3]. As a result of increased use of carbapenems, carbapenemases are widespread in Enterobacteriaceae family and particularly in Klebsiella pneumoniae; so effective treatment options are decreasing $[2,4]$. Carbapenemase-producing K. pneumoniae emerged in the late 1990s and has become a serious health problem worldwide [5].

Carbapenem-resistant K. pneumoniae (CRKP) can cause nosocomial infections and outbreaks with high mortality rates. Such infections occur mainly in patients admitted to intensive care units (ICU) with several underlying diseases and histories of having received prolonged courses of antibiotics [6]. Several resistance mechanisms have been described. Carbapenemase production (K. pneumoniae carbapenemase [KPC]), metallo- $\beta$-lactamase (MBL) and porin loss, combined with the overproduction of ESBL, are described as the most common mechanisms of carbapenem resistance $[7,8]$

Carbapenemases producing $K$. pneumoniae strains are considered endemic in some areas. For example, studies by the European Antimicrobial Resistance Surveillance (EARS-net) showed that the prevalence of carbapenem-resistant $K$. pneumoniae has increased from $1-2 \%$ to $15 \%$ in Italy in 2006-2009 [9]. Due to this resistance spreading rapidly around the world, there has been a need for new therapeutic agents. Polymyxin and colistin were out of use since the early 1970 s due to its side effects of neurotoxicity and nephrotoxicity, but colistin has become a preferred antimicrobial agent with increase of infections with resistant Enterobacteriaceae [10-12]. However, excessive use of colistin has led to resistance to this drug [13]. The resistance to colistin seen in $K$. pneumoniae strains is reported to be due to reduced affinity of colistin to lipopolysaccharide target [5]. In this study, colistin resistance in CRKP strains has been identified and evaluated.

Since there are not many studies with determination of colistin minimum inhibitory concentration (MIC), we undertook this study to estimate and evaluate colistin resistance in our hospital by MIC.

\section{MATERIALS AND METHODS}

The present study was carried out in a Tertiary Care Hospital of Karnataka, South India, with bed strength of 650 . A total of 518 nonrepetitive clinical isolates of $K$. pneumoniae were collected over a period of 3 years (2014-2016) from our ICUs, i.e. multidisciplinary ICU (MICU), neurosurgery ICU, intensive thoracic unit, neonatal ICU, pediatric ICU, coronary care unit, and renal ICU. These isolates were obtained from endotracheal aspirate (111), blood (80), urine (59), pus (22), bronchoalveolar lavage and sputum (21), catheter tips (15), fluids (13), and tissue specimens (8).

K. pneumoniae was identified using standard biochemical methods Disk-diffusion tests were performed to determine susceptibility to antimicrobials, according to the Clinical and Laboratory Standards Institute (CLSI). Escherichia coli ATCC 25922 was used as a quality control. Isolates were screened for the ESBL phenotype by the standard double-disk synergy test, and KPC was screened both by evaluating breakpoints and using modified Hodge test, according to CLSI guidelines [14]. 
Imipenem plus ethylenediaminetetraacetic acid (EDTA) discs were used to detect the presence of MBL (HiMedia Laboratories, Mumbai). MICs of meropenem and colistin (Sigma-Aldrich Corporation, St. Louis, US) were determined by the agar dilution method according to the guidelines from the CLSI [14]. The colistin breakpoint was evaluated using breakpoints for Enterobacteriaceae recommended by the European Committee on Antibiotic Susceptibility Testing. (resistant: $>2 \mathrm{ug} / \mathrm{ml}$; sensitive: $\leq 2 \mathrm{ug} / \mathrm{ml}$ ). K. pneumoniae ATCC 700603 was used as a quality control.

\section{RESULTS}

Totally 518 isolates of $K$. pneumoniae were isolated during the study, of which 329 (63.5\%) isolates were CRKP isolates. The clinical source and distribution of $K$. pneumoniae in different ICUs are shown in Tables 1 and 2 .

The antibiotic susceptibility of CRKP to other drugs is shown in Table 3. Antibiotic susceptibility testing revealed that very few antibiotics were susceptible.

With regard to colistin $91 / 329$ (27.65\%), isolates were resistant and the MIC ranged between 4 and $>512 \mathrm{ug} / \mathrm{ml} \mathrm{MIC}_{90}$ and $\mathrm{MIC}_{50}$ of colistin were 16 and $4 \mathrm{ug} / \mathrm{ml}$ (Fig. 1).

In the present study, we found that 85/91 (93.4\%) isolates that showed resistance to colistin were from MICU and resistance to carbapenems also seen in MICU (83.3\%).

Table 1: Clinical sources of carbapenem-resistant Klebsiella pneumoniae (329)

\begin{tabular}{ll}
\hline Clinical specimen & N (\%) \\
\hline Tracheal aspirate & $111(33.73)$ \\
Blood & $80(24.3)$ \\
Urine & $59(17.9)$ \\
Pus & $22(6.7)$ \\
BAL and sputum & $21(6.4)$ \\
Catheter tips & $15(4.5)$ \\
Fluids & $13(3.95)$ \\
Tissue & $8(2.4)$ \\
\hline
\end{tabular}

Table 2: Distribution of CRKP in ICUs

\begin{tabular}{ll}
\hline ICU & $\mathbf{N}(\%)$ \\
\hline MICU & $274(83.3)$ \\
NSICU & $25(7.9)$ \\
ITU & $11(3.34)$ \\
NICU & $8(2.43)$ \\
PICU & $5(1.5)$ \\
CCU & $3(0.91)$ \\
RICU & $2(0.60)$ \\
\hline
\end{tabular}

ICU: Intensive care unit, MICU: Multidisciplinary intensive care unit, NSICU: Neurosurgery intensive care unit, ITU: Intensive thoracic unit, NICU: Neonatal intensive care unit, PICU: Pediatric intensive care unit, CCU: Critical care unit, RICU: Renal intensive care unit, CRKP: Carbapenem-resistant K. pneumoniae

Table 3: Antibiotic susceptibility pattern of CRKP* $(n=329)$

\begin{tabular}{ll}
\hline Antibiotic & CRKP (\%) susceptible \\
\hline Cotrimoxazole & $22(6.6)$ \\
Gentamicin & $58(17.6)$ \\
Amikacin & $59(17.9)$ \\
Netilimicin & $42(12.7)$ \\
Tobramicin & $19(5.7)$ \\
Ciprofloxacin & $9(2.7)$ \\
Ofloxacin & $10(3.0)$ \\
Levofloxacin & $22(6.6)$ \\
\hline
\end{tabular}

*All cephalosporins, betalacatamase, and betalactamase inhibitors were resistant. CRKP: Carbapenem-resistant Klebsiella pneumoniae
Out of 329 carbapenem-resistant isolates, 216 were positive for modified-Hodge test and $59 \mathrm{~K}$. pneumoniae were screened positive for imipenem/imipennem EDTA test. The rest of the 54 carbapenemresistant isolates were negative for both tests.

\section{DISCUSSION}

K. pneumoniae is highly prevalent in hospitals and causes many nosocomial infections. The emergence of drug resistance in $K$. pneumoniae continues to be of critical concern for the choice of treatment options against infections caused by this bacterium.

Carbapenem resistance in Klebsiella spp. is an emerging problem and is a cause of concern as many nosocomial Klebsiella spp. are detected to be resistant to most other antibiotics.

Colistin is used as last resort of antimicrobials, especially in the present worrisome therapeutic scenario of multidrug resistant and pan drug-resistant Gram-negative infections. The drug acts on outer cell membrane of Gram-negative bacteria (GNB) and releases lipopolysaccharides [15]. This in turn results into disruption of cell membrane leading to leakage of cell content, causing cell lysis and finally cell death $[16,17]$. Within years after the reuse of colistin, there have been reports of colistin-resistant strains [18]. Indiscriminate antibiotic use in India is leading to cases of bacteria resistant to colistin.

ICUs are the epicenter for spawning multidrug resistance within hospitals. Many patients are transferred to the ICU from other healthcare facilities, where they have acquired resistant pathogens. Patients within the ICU undergo invasive procedures, treatment with antibiotic

Combinations and exposure to other patients with resistant pathogens [19]. Multiple mechanisms exist for ICU pathogens to acquire antibiotic resistance. These mechanisms include enzymatic inhibition of drugs, alteration of proteins targeted by antibiotics, changes in metabolic pathways, antibiotic efflux, alterations in porin channels, and changes of membrane permeability [20]

In the present study, we found $63.5 \%$ (329/518) K. pneumoniae was multidrug resistant. The majority of the carbapenem resistant isolates were isolated form tracheal aspirate $(33.73 \%)$, followed by blood $(24.3 \%)$ and urine (17.9\%). Gupta et al. found resistance to meropenem $(22.16 \%)$ was more compared to imipenem $(17.32 \%)$ in his study from different bacteria

Healso found that a significantly high resistance was seen in ICU patients, $37.3 \%$ and $31.9 \%$ for meropenem and imipenem, respectively [21]. El-Mahdy et al. in his work on cancer patients found only $4 \%$ of them were resistant to carbapenems [22]. Bashir et al. in his research study found $27.9 \%$ isolates were carbapenem-resistant [23].

Dizbay et al. [24] analyzed nosocomial infections produced by carbapenem-resistant Klebsiella spp. in ICU and their risk factors. They found that carbapenem resistance is significantly high in ICUs and more

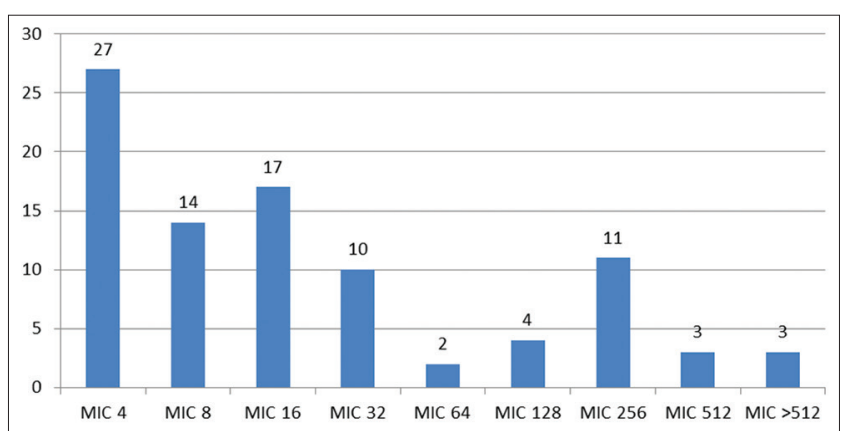

Fig. 1: Minimum inhibitory concentration (MIC) of colistin. MIC90 - $16 \mathrm{ug} / \mathrm{ml}$ and MIC50 - $4 \mathrm{ug} / \mathrm{ml}$ 
frequent $(78.57 \%)$, and resistance was more often seen in respiratory tract specimens.

In the current study, all the isolates were screened for MIC of colistin. The prevalence of colistin resistance in $K$. pneumoniae from ICUs was (91/329) 27.65\%, and the MIC ranged between 4 and $>512 \mathrm{ug} / \mathrm{ml}$.

Taneja et al. in a study from North India found $16 \%$ of the carbapenemresistant strains were resistant to both tigecycline and colistin [25]. Giani et al. in Italy conducted a nationwide cross-sectional survey on CRE, carried out in mid-2011, the overall percentage of colistin resistance among KPC-KP was found to be $22.4 \%$ [26]. In another study in Italy Capone et al. observed 36.1\% resistance to colistin in CRKP [18]. In a recent study by Kontopidou et al., 51 (34\%) patients were colonized by pathogens with an intrinsic resistance to colistin [13].

Ghafur et al. reported a series of 13 patients with colistin resistance from South India [27].

With the increased emergence and spread of CRKP, treatment options are dcecreasing. As a reserve agent, colistin has been the drug of choice in the treatment of CRKP, but with the rise of carbapenem resistance, the colistin usage has been increased over the years leading to slow emergence of colistin resistance. Especially, if these antibiotics are not used in combinations, they are not enough as therapeutic agent's leads to treatment failure and high mortality rates. The high rate of resistance to colistin and carbapenems is worrisome, but the MIC of colistin and carbapenems helps the clinician to choose the drug in appropriate combinations.

Shah et al. conducted a study in a tertiary care hospital in Mumbai, the purpose of this study was to evaluate the efficacy of colistin-carbapenem combination against Carbapenem-resistant GNB (CRGNB) infection in a clinical study and an in vitro synergy study using Etest. Overall, 60.6\% clinical success was observed in patients receiving colistin-carbapenem combination against CRGNB infection [28].

Tumbarello et al. [29] in his study emphasized the importance of combination therapy in carbapenemase-producing Klebsiella pneumonia. Combination therapy with tigecycline, colistin, and meropenem (MIC upto $8 \mathrm{ug} / \mathrm{ml}$ ) was associated with lower mortality.

In another study by Daikos et al. [30] stated that combination therapy with carbapenem MIC of 8 was more beneficiary in severely ill patients by lowering the mortality rate.

\section{CONCLUSION}

The present study showed the emergence of colistin resistance among clinical isolates of $K$. pneumoniae which are alarming in ICUs. It is important to determine the MIC of colistin to formulate an effective treatment protocol, even for the resistant isolates. For the resistant cases, colistin should be used in combination with other antimicrobials for therapy. Necessary infection control precautions and increased awareness to prevent further rise in the drug resistance against this last resort of antimicrobials is important. A restricted and rational use of the colistin is the need of hour.

\section{REFERENCES}

1. Perçin D, Colakoğlu S, Durmaz S, Ekincioğlu P. Comparison of ertapenem-EMB Agar with traditional methods for screening carbapenem-resistant $K$. pneumoniae from rectal swabs. Mikrobiyol Bul 2012;46:546-52.

2. Poirel L, Labarca J, Bello H, Rioseco ML, Bernabeu S, Nordmann P. Emergence of the $16 \mathrm{~S}$ rRNA methylase RmtG in an extended-spectrumlactamase-producing and colistin-resistant $K$. pneumoniae isolate in chile. Antimicrob Agents Chemother 2014;58:618-9.

3. Kalem F, Ergun AG, Ertuğrul O, Özçimen S, Şimşek H, Süzük S, et al. Colistin resistance in carbapenem-resistant Klebsiella pneumoniae strains. Biomed Res 2016;27(2):368-72.
4. Nordmann P, Naas T, Poirel L. Global spread of carbapenemaseproducing Enterobacteriaceae. Emerg Infect Dis 2011;17(10):1791-8.

5. Cannatelli A, D'Andrea MM, Giani T, Di Pilato V, Arena F, Ambretti S, et al. In vivo emergence of colistin resistance in Klebsiella pneumoniae producing KPC-type carbapenemases mediated by insertional inactivation of the $\mathrm{PhoQ} / \mathrm{PhoP}$ mgrB regulator. Antimicrob Agents Chemother 2013;57(11):5521-6.

6. Maltezou HC. Metallo-beta-lactamases in gram-negative bacteria: Introducing the era of pan-resistance? Int $\mathrm{J}$ Antimicrob Agents 2009;33(5):405.e1-7.

7. Peirano G, Seki LM, Val Passos VL, Pinto MC, Guerra LR, Asensi MD. Carbapenem-hydrolysing beta-lactamase KPC-2 in Klebsiella pneumoniae isolated in Rio de Janeiro, Brazil. J Antimicrob Chemother 2009;63(2):265-8.

8. Souli M, Kontopidou FV, Papadomichelakis E, Galani I, Armaganidis A, Giamarellou H. Clinical experience of serious infections caused by Enterobacteriaceae producing VIM-1 metallo-beta-lactamase in a Greek University Hospital. Clin Infect Dis 2008;46(6):847-54.

9. Mammina C, Bonura C, Di Bernardo F, Aleo A, Fasciana T, Sodano $\mathrm{C}$, et al. Ongoing spread of colistin-resistant Klebsiella pneumoniae in different wards of an acute general hospital, Italy, June to December 2011. Euro Surveill 2012;17(33). pii:20248

10. Garbati MA, Bin Abdulhak A, Baba K, Sakkijha H. Infection due to colistin-resistant Enterobacteriacae in critically-ill patients. J Infect Dev Ctries 2013;7(10):713-9.

11. Bogdanovich T, Adams-Haduch JM, Tian GB, Nguyen MH, Kwak EJ, Muto CA, Doi Y. Colistin-resistant, Klebsiella pneumoniae carbapenemase [KPC]-producing Klebsiella pneumoniae belonging to the international epidemic clone ST258. Clin Infect Dis 2011;53(4):373-6.

12. Cannatelli A, Giani T, D’Andrea MM, Di Pilato V, Arena F, Conte V, et al. MgrB inactivation is a common mechanism of colistin resistance in KPC-producing Klebsiella pneumoniae of clinical origin. Antimicrob Agents Chemother 2014;58(10):5696-703.

13. Kontopidou F, Plachouras D, Papadomichelakis E, Koukos G, Galani I, Poulakou $\mathrm{G}$, et al. Colonization and infection by colistin-resistant gramnegative bacteria in a cohort of critically ill patients. Clin Microbiol Infect 2011;17(11):E9-11.

14. CLSI. Performance Standards for Antimicrobial Susceptibility Testing; Twenty-First Informational Supplement. CLSI document M100-S21. Wayne, PA: Clinical and Laboratory Standards Institute; 2011.

15. Cai Y, Chai D, Wang R, Liang B, Bai N. Colistin resistance of Acinetobacter baumannii: Clinical reports, mechanisms and antimicrobial strategies. J Antimicrob Chemother 2012;67(7):1607-15.

16. Falagas ME, Kasiakou SK, Tsiodras S, Michalopoulos A. The use of intravenous and aerosolized polymyxins for the treatment of infections in critically ill patients: A review of the recent literature. Clin Med Res 2006;4(2):138-46

17. Li J, Nation RL, Milne RW, Turnidge JD, Coulthard K. Evaluation of colistin as an agent against multi-resistant gram-negative bacteria. Int $\mathrm{J}$ Antimicrob Agents 2005;25(1):11-25.

18. Capone A, Giannella M, Fortini D, Giordano A, Meledandri M, Ballardini M, et al. High rate of colistin resistance among patients with carbapenem-resistant Klebsiella pneumoniae infection accounts for an excess of mortality. Clin Microbiol Infect 2013;19(1):E23-30.

19. Lucet JC, Decre D, Fichelle A, Joly-Guillou ML, Pernet M, Deblangy C. Control of a prolonged outbreak of extended-spectrum $\beta$-lactamaseproducing Enterobacteriaceae in a University hospital. Clin Infect Dis 1999;29(6):1411-8.

20. Virk A, Steckelberg JM. Clinical aspects of antimicrobial resistance. Mayo Clin Proc 2000;75(2):200-14.

21. Gupta E, Mohanty S, Sood S, Dhawan B, Das BK, Kapil A. Emerging resistance to carbapenems in a tertiary care hospital in north India. Indian J Med Res 2006;124:95-8

22. El-Mahdy TS, Abdelaziz MO, El-Domany RA. Prevalence and molecular characterization of extended spectrum $\beta$-lacatamases in Klebsiella pneumoniae isolates from cancer patients and others. Int J Pharm Pharm Sci 2015;7:122-7.

23. Bashir H, Kakru KD, Qadri SS, Bali N, Bshir S, Ione S. Carbapenem resistant Klebsiella pneumoniae in a tertiary care hospital in North India. Int J Adv Res 2014;2(1):92-102.

24. Dizbay M, Tunccan GO, Karasahin O, Aktas F. Emergence of carbapenem resistant Klebsiella spp. infections in a Turkish university hospital: Epidemiology and risk factors. J Infect Dev Ctries 2014;8(1):44-9.

25. Taneja N, Singh G, Singh M, Sahrms M. Emergence of tigecycline 
\& colistin resistant Acinetobacter baumanii in patients with complicated urinary tract infections in North India. Indian J Med Res 2011;133(6):681-4.

26. Giani T, Pini B, Arena F, Conte V, Bracco S, Migliavacca R; AMCLI-CRE Survey Participants, et al. Epidemic diffusion of KPC carbapenemase-producing Klebsiella pneumoniae in Italy: Results of the first countrywide survey, 15 May to 30 June 2011. Euro Surveill 2013;18. pii:20489.

27. Ghafur A, Vidyalakshmi PR, Murali A, Priyadarshini K, Thirunarayan MA. Emergence of pan-drug resistance amongst gram negative bacteria! The first case series from India. J Microbiol Infect Dis 2014;4(3):86-91

28. Shah PG, Shah SR, Kamat SD, Kamat DV. Colistin-carbapenem combination therapy against carbapenem resistant gram negative bacilli infections: Clinical and in vitro synergy study. Int J Pharm Pharm Sci 2014;6:497-500.

29. Tumbarello M, Viale P, Viscoli C, Trecarichi EM, Tumietto F, Marchese A, et al. Predictors of mortality in bloodstream infections caused by Klebsiella pneumoniae carbapenemase-producing $K$. pneumoniae: Importance of combination therapy. Clin Infect Dis 2012;55(7):943-50.

30. Daikos GL, Tsaousi S, Tzouvelekis LS, Anyfantis I, Psichogiou M, Argyropoulou A, et al. Carbapenemase-producing Klebsiella pneumoniae bloodstream infections: Lowering mortality by antibiotic combination schemes and the role of carbapenems. Antimicrob Agents Chemother 2014;58(4):2322-8. 